\title{
Existence and concentration of solution for a non-local regional Schrödinger equation with competing potentials
}

\author{
Claudianor O. Alves \\ Universidade Federal de Campina Grande \\ Unidade Acadêmica de Matemática \\ CEP: 58429-900 - Campina Grande - PB, Brazil \\ coalves@mat.ufcg.edu.br \\ César E. Torres Ledesma \\ Departamento de Matemáticas, \\ Universidad Nacional de Trujillo, \\ Av. Juan Pablo II s/n. Trujillo-Perú \\ ctl_576@yahoo.es
}

\begin{abstract}
In this paper, we study the existence and concentration phenomena of solutions for the following non-local regional Schrödinger equation

$$
\left\{\begin{array}{l}
\epsilon^{2 \alpha}(-\Delta)_{\rho}^{\alpha} u+Q(x) u=K(x)|u|^{p-1} u, \text { in } \mathbb{R}^{n} \\
u \in H^{\alpha}\left(\mathbb{R}^{n}\right)
\end{array}\right.
$$

where $\epsilon$ is a positive parameter, $0<\alpha<1,1<p<\frac{n+2 \alpha}{n-2 \alpha}, n>2 \alpha ;(-\Delta)_{\rho}^{\alpha}$ is a variational version of the regional fractional Laplacian, whose range of scope is a ball with radius $\rho(x)>0, \rho, Q, K$ are competing functions. We study the existence of ground state and we analyze the behavior of semiclassical solutions as $\epsilon \rightarrow 0$.
\end{abstract}

\section{INTRODUCTION}

The aim of this article is to study the non-linear Schrödinger equation with non-local regional diffusion and competing potentials

$$
\left\{\begin{aligned}
\epsilon^{2 \alpha}(-\Delta)_{\rho}^{\alpha} u+Q(x) u & =K(x)|u|^{p-1} u, \text { in } \mathbb{R}^{n}, \\
u & \in H^{\alpha}\left(\mathbb{R}^{n}\right),
\end{aligned}\right.
$$

where $0<\alpha<1, \epsilon>0, n>2 \alpha, Q, K \in C\left(\mathbb{R}^{n}, \mathbb{R}^{+}\right)$are bounded and the operator $(-\Delta)_{\rho}^{\alpha}$ is a variational version of the non-local regional fractional Laplacian, with range of scope determined by a positive function $\rho \in C\left(\mathbb{R}^{n}, \mathbb{R}^{+}\right)$, which is defined as

$$
\int_{\mathbb{R}^{n}}(-\Delta)_{\rho}^{\alpha} u(x) \varphi(x) d x=\int_{\mathbb{R}^{n}} \int_{B(0, \rho(x))} \frac{[u(x+z)-u(z)][\varphi(x+z)-\varphi(x)]}{|z|^{n+2 \alpha}} d z d x
$$


In what follows, we will work with the problem

$$
(-\Delta)_{\rho_{\epsilon}}^{\alpha} v+Q(\epsilon x) v=K(\epsilon x)|v|^{p-1} v, \quad x \in \mathbb{R}^{n},
$$

with and $\rho_{\epsilon}=\frac{1}{\epsilon} \rho(\epsilon x)$, which is equivalent to $(P)$ by considering the change variable $v(x)=u(\epsilon x)$.

Associated with $\left(P^{\prime}\right)$ we have the energy functional $I_{\rho_{\epsilon}}: X^{\epsilon} \rightarrow \mathbb{R}$ defined as

$$
\begin{aligned}
I_{\rho_{\epsilon}}(v)= & \frac{1}{2}\left(\int_{\mathbb{R}^{n}} \int_{B\left(0, \frac{1}{\epsilon} \rho(\epsilon x)\right)} \frac{|v(x+z)-v(x)|^{2}}{|z|^{n+2 \alpha}}+\int_{\mathbb{R}^{n}} Q(\epsilon x)|v(x)|^{2} d x\right)- \\
& \frac{1}{p+1} \int_{\mathbb{R}^{n}} K(\epsilon x)|v(x)|^{p+1} d x,
\end{aligned}
$$

where $X^{\epsilon}$ denotes the Hilbert space $H^{\alpha}\left(\mathbb{R}^{n}\right)$ endowed with the norm

$$
\|v\|_{\rho_{\epsilon}}=\left(\int_{\mathbb{R}^{n}} \int_{B\left(0, \rho_{\epsilon}(x)\right)} \frac{|v(x+z)-v(x)|^{2}}{|z|^{n+2 \alpha}} d z d x+\int_{\mathbb{R}^{n}} Q(\epsilon x)|v(x)|^{2} d x\right)^{\frac{1}{2}} .
$$

Hereafter, we say that $v \in X^{\epsilon}$ is a weak solution of $\left(P^{\prime}\right)$ if $v$ is a critical point of $I_{\rho_{\epsilon}}$. In Section 2, Proposition 2.1, it is proved that \|\|$_{\rho_{\epsilon}}$ is equivalent to the usual norm in $H^{\alpha}\left(\mathbb{R}^{n}\right)$.

Recently, the study on problems of fractional Schrödinger equations has attracted much attention from many mathematicians. In the case of the fractional Laplacian $(-\Delta)^{\alpha}$, Chen [3] studied the existence of ground sate solution of nonlinear fractional Schrödinger equation

$$
(-\Delta)^{\alpha} u+V(x) u=u^{p} \text { in } \mathbb{R}^{n}
$$

with unbounded potential. The existence of a ground state of (1.2) is obtained by a Lagrange multiplier method and the Nehari manifold method is used to obtain standing waves with prescribed frequency. If $V(x)=1$, Dipierro et al. 6] proved existence and symmetry results for the solution of equation (1.2). Felmer et al. [8], studied the same equation with a more general nonlinearity $f(x, u)$, they obtained the existence, regularity and qualitative properties of ground states. Secchi [14] obtained positive solutions of a more general fractional Schrödinger equation by the variational method.

On the other hand, research has been done in recent years regarding regional fractional Laplacian, where the scope of the operator is restricted to a variable region near each point. We mention the work by Guan [11] and Guan and Ma [12] where they study these operators, their relation with stochastic processes and they develop integration by parts formula, and the work by Ishii and Nakamura [13], where the authors studied the Dirichlet problem for regional fractional Laplacian modeled on the p-Laplacian. 
Recently, Felmer and Torres [9, 10] considered positive solution of nonlinear Schrödinger equation with non-local regional diffusion

$$
\epsilon^{2 \alpha}(-\Delta)_{\rho}^{\alpha} u+u=f(u), \quad u \in H^{\alpha}\left(\mathbb{R}^{n}\right),
$$

where the operator $(-\Delta)_{\rho}^{\alpha}$ is defined as above. Under suitable assumptions on the non-linearity $f$ and the range of scope $\rho$, they obtained the existence of a ground state by mountain pass argument and a comparison method. Furthermore, they analyzed symmetry properties and concentration phenomena of these solutions. These regional operators present various interesting characteristics that make them very attractive from the point of view of mathematical theory of non-local operators. We also mention the recent works by Torres [17, 18, 19], where existence, multiplicity and symmetry results are considered in bounded domain and $\mathbb{R}^{n}$.

We recall that when $(-\Delta)_{\rho}^{\alpha}$ is replaced by $(-\Delta)^{\alpha}$, Chen and Zheng [4] studied (1.3) with external potential $V(x)$ and $f(u)=|u|^{p-1} u$. They showed that when $n=1,2,3, \epsilon$ is sufficiently small, $\max \left\{\frac{1}{2}, \frac{n}{4}\right\}<\alpha<1$ and $V$ satisfies some smoothness and boundedness assumptions, equation (1.3) has a nontrivial solution $u_{\epsilon}$ concentrated to some single point as $\epsilon \rightarrow 0$. Very recently, in [5], Dávila, del Pino and Wei generalized various existence results known for (1.3) with $\alpha=1$ to the case of fractional Laplacian. Moreover, we also mention the works by Shang and Zhang [15, 16, where it was considered the nonlinear fractional Schrödinger equation with competing potentials

$$
\epsilon^{2 \alpha}(-\Delta)^{\alpha} u+V(x) u=K(x)|u|^{p-2} u+Q(x)|u|^{q-2} u, \quad x \in \mathbb{R}^{n},
$$

where $2<q<p<2_{\alpha}^{*}$. By using perturbative variational method, mountain pass arguments and Nehari manifold method, they analyzed the existence, multiplicity and concentration phenomena for the solutions of (1.4).

Motivated by these previous works, in this paper, our goal is to study the existence and concentration phenomena for the solutions of $(P)$. As pointed out in [1, 2, 20], the geometry of the ground state energy function $C(\xi)$, which is defined to be the ground state level associated with

$$
(-\Delta)^{\alpha} u+Q(\xi) u=K(\xi)|u|^{p-1} u, \quad x \in \mathbb{R}^{n},
$$

where $\xi \in \mathbb{R}^{n}$ is regard as a parameter instead of an independent variable, it is crucial in our approach. Here, the functions $\rho, Q$ and $K$ satisfy the following conditions:

$\left(H_{0}\right)$ There are positive real numbers $Q_{\infty}, K_{\infty}$ such that

$$
Q_{\infty}=\lim _{|\xi| \rightarrow+\infty} Q(\xi) \text { and } K_{\infty}=\lim _{|\xi| \rightarrow+\infty} K(\xi) .
$$

$\left(H_{1}\right)$ There are numbers $0<\rho_{0}<\rho_{\infty} \leq \infty$ such that

$$
\rho_{0} \leq \rho(\xi)<\rho_{\infty}, \quad \forall \xi \in \mathbb{R}^{n} \quad \text { and } \quad \lim _{|\xi| \rightarrow \infty} \rho(\xi)=\rho_{\infty} .
$$

$\left(H_{2}\right) Q, K: \mathbb{R}^{n} \rightarrow \mathbb{R}$ are continuous function satisfying

$$
0<a_{1} \leq Q(\xi), K(\xi) \leq a_{2} \quad \forall \xi \in \mathbb{R}^{n}
$$


for some positive constants $a_{1}, a_{2}$.

Theorem 1.1. Assume $\left(H_{0}\right)-\left(H_{2}\right)$. Then, if

$$
\inf _{\xi \in \mathbb{R}^{n}} C(\xi)<\liminf _{|\xi| \rightarrow+\infty} C(\xi),
$$

problem $(P)$ has a ground state solution $u_{\epsilon} \in X^{\epsilon}$ for $\epsilon$ small enough. Moreover, for each sequence $\epsilon_{m} \rightarrow 0$, there is a subsequence such that for each $m \in \mathbb{N}$, the solution $u_{\epsilon_{m}}$ concentrates around a minimum point $\xi^{*}$ of the function $C(\xi)$, in the following sense: given $\delta>0$, there are $\epsilon_{0}, R>0$ such that

$\int_{B^{c}\left(\xi^{*}, \epsilon_{m} R\right)}\left|u_{\epsilon_{m}}\right|^{2} d x \leq \epsilon_{m}^{n} \delta \quad$ and $\quad \int_{B\left(\xi^{*}, \epsilon_{m} R\right)}\left|u_{\epsilon_{m}}\right|^{2} d x \geq \epsilon_{m}^{n} C, \quad \forall \epsilon_{m} \leq \epsilon_{0}$, where $C$ is a constant independent of $\delta$ and $m$.

We would like to point out that the condition $(C)$ is not empty, because it holds by supposing that there is $\xi_{0} \in \mathbb{R}^{n}$ such that

$$
\frac{Q\left(\xi_{0}\right)^{\frac{p+1}{p-1}-\frac{n}{2 \alpha}}}{K\left(\xi_{0}\right)^{\frac{2}{p-1}}}<\frac{Q_{\infty}^{\frac{p+1}{p-1}-\frac{n}{2 \alpha}}}{K_{\infty}^{\frac{2}{p-1}}} .
$$

For more details, see Corollary 3.1] in Section 3.

\section{Preliminaries}

In this section we recall some basic facts about the Sobolev space $H^{\alpha}\left(\mathbb{R}^{n}\right)$ such as embeddings and compactness properties. To begin with, we recall the following embedding theorem.

Theorem 2.1. ([7]) Let $\alpha \in(0,1)$, then there exists a positive constant $C=C(n, \alpha)$ such that

$$
\|u\|_{L^{2_{\alpha}^{*}\left(\mathbb{R}^{n}\right)}}^{2} \leq C \int_{\mathbb{R}^{n}} \int_{\mathbb{R}^{n}} \frac{|u(x)-u(y)|^{2}}{|x-y|^{n+2 \alpha}} d y d x
$$

and then $H^{\alpha}\left(\mathbb{R}^{n}\right) \hookrightarrow L^{q}\left(\mathbb{R}^{n}\right)$ is continuous for all $q \in\left[2,2_{\alpha}^{*}\right]$. Moreover, $H^{\alpha}\left(\mathbb{R}^{n}\right) \hookrightarrow L^{q}(\Omega)$ is compact for any bounded set $\Omega \subset \mathbb{R}^{n}$ and for all $q \in$ $\left[2,2_{\alpha}^{*}\right)$, where $2_{\alpha}^{*}=\frac{2 n}{n-2 \alpha}$ is the critical exponent.

The next lemma establishes that \|\|$_{\rho_{\epsilon}}$ is equivalent to usual norm in $H^{\alpha}\left(\mathbb{R}^{n}\right)$.

Proposition 2.1. Suppose that $\left(H_{1}\right)$ and $\left(H_{2}\right)$ hold and set

$$
\|u\|=\left(\int_{\mathbb{R}^{n}} \int_{\mathbb{R}^{n}} \frac{|u(x)-u(z)|^{2}}{|x-z|^{n+2 \alpha}} d z d x+\int_{\mathbb{R}^{n}}|u(x)|^{2} d x\right)^{\frac{1}{2}}
$$

the usual norm in $H^{\alpha}\left(\mathbb{R}^{n}\right)$. Then, there exists a constant $\mathfrak{S}>0$ independent of $\epsilon$ such that

$$
\|u\| \leq \mathfrak{S}\|u\|_{\rho_{\epsilon}}, \quad \forall u \in H^{\alpha}\left(\mathbb{R}^{n}\right) .
$$

From this, $\|\cdot\|$ and $\|\cdot\|_{\rho_{\epsilon}}$ are equivalents norms in $H^{\alpha}\left(\mathbb{R}^{n}\right)$. 
Proof. Without loss of generality we will consider $\epsilon=1$. For $u \in X^{1}=$ $H^{\alpha}\left(\mathbb{R}^{n}\right)$, the Fubini's Theorem together with $\left(H_{1}\right)$ and $\left(H_{2}\right)$ gives

$$
\begin{aligned}
& a_{1}\|u\|^{2}=a_{1} \int_{\mathbb{R}^{n}}|u(x)|^{2} d x+a_{1} \int_{\mathbb{R}^{n}} \int_{B\left(x, \rho_{0}\right)} \frac{|u(x)-u(z)|^{2}}{|x-z|^{n+2 \alpha}} d z d x+ \\
& \quad a_{1} \int_{\mathbb{R}^{n}} \int_{B^{c}\left(x, \rho_{0}\right)} \frac{|u(x)-u(z)|^{2}}{|x-z|^{n+2 \alpha}} d z d x \\
& \leq\left(1+\frac{2\left|S^{n-1}\right|}{\alpha \rho_{0}^{2 \alpha}}\right) \int_{\mathbb{R}^{n}} Q(x)|u(x)|^{2} d x+a_{1} \int_{\mathbb{R}^{n}} \int_{B\left(x, \rho_{0}\right)} \frac{|u(x)-u(z)|^{2}}{|x-z|^{n+2 \alpha}} d z d x \\
& \leq A\left(\int_{\mathbb{R}^{n}} Q(x)|u(x)|^{2} d x+\int_{\mathbb{R}^{n}} \int_{B(0, \rho(x))} \frac{|u(x+z)-u(x)|^{2}}{|z|^{n+2 \alpha}} d z d x\right),
\end{aligned}
$$

where $A=\max \left\{a_{1},\left(1+\frac{2\left|S^{n-1}\right|}{\alpha \rho_{0}^{2 \alpha}}\right)\right\}$. The proposition follows by taking $\mathfrak{S}=\frac{1}{a_{1}} A$.

The following lemma is a version of the concentration compactness principle proved by Felmer and Torres [9], which will be use later on.

Lemma 2.1. Let $n \geq 2$. Assume that $\left\{u_{k}\right\}$ is bounded in $H_{\rho}^{\alpha}\left(\mathbb{R}^{n}\right)$ and it satisfies

$$
\lim _{k \rightarrow \infty} \sup _{y \in \mathbb{R}^{n}} \int_{B(y, R)}\left|u_{k}(x)\right|^{2} d x=0,
$$

where $R>0$. Then $u_{k} \rightarrow 0$ in $L^{q}\left(\mathbb{R}^{n}\right)$ for $2<q<2_{\alpha}^{*}$.

\section{Ground state}

We prove the existence of weak solution of $\left(P^{\prime}\right)$ finding a critical point of the functional $I_{\rho_{\epsilon}}$. Using the embeddings given in Theorem 2.1, it follows that the functional $I_{\rho_{\epsilon}}$ is of class $C^{1}\left(X^{\epsilon}, \mathbb{R}\right)$ with

$$
I_{\rho_{\epsilon}}^{\prime}(u) v=\langle u, v\rangle_{\rho_{\epsilon}}-\int_{\mathbb{R}^{n}} K(\epsilon x)|u(x)|^{p-1} u(x) v(x) d x, \quad \forall v \in X^{\epsilon}
$$

where

$\langle u, v\rangle_{\rho_{\epsilon}}=\int_{\mathbb{R}^{n}} \int_{B\left(0, \rho_{\epsilon}(x)\right)} \frac{[u(x+z)-u(x)][v(x+z)-v(x)]}{|z|^{n+2 \alpha}} d z d x+\int_{\mathbb{R}^{n}} Q(\epsilon x) u v d x$.

Using well known arguments, it follows that $I_{\rho_{\epsilon}}$ verifies the mountain pass geometry. Then, there is a $(P S)_{c}$ sequence $\left\{u_{k}\right\} \subset X^{\epsilon}$ such that

$$
I_{\rho_{\epsilon}}\left(u_{k}\right) \rightarrow C_{\rho_{\epsilon}} \text { and } I_{\rho_{\epsilon}}^{\prime}\left(u_{k}\right) \rightarrow 0
$$

where $C_{\rho_{\epsilon}}$ is the mountain pass level given by

$$
C_{\rho_{\epsilon}}=\inf _{\gamma \in \Gamma{ }_{\rho_{\epsilon}} \sup _{t \in[0,1]}} I_{\rho_{\epsilon}}(\gamma(t))>0
$$

with

$$
\Gamma_{\rho_{\epsilon}}=\left\{\gamma \in C\left([0,1], X^{\epsilon}\right): \gamma(0)=0, \quad I_{\rho_{\epsilon}}(\gamma(1))<0\right\}
$$


In the sequel, $\mathcal{N}_{\rho_{\epsilon}}$ denotes the Nehari manifold associated to the functional $I_{\rho_{\epsilon}}$, that is,

$$
\mathcal{N}_{\rho_{\epsilon}}=\left\{u \in X^{\epsilon} \backslash\{0\}: I_{\rho_{\epsilon}}^{\prime}(u) u=0\right\} .
$$

It is easy to see that all non trivial solutions of $\left(P^{\prime}\right)$ belongs to $\mathcal{N}_{\rho_{\epsilon}}$. Moreover, by using standard arguments, it is possible to prove that

$$
C_{\rho_{\epsilon}}=\inf _{u \in \mathcal{N}_{\rho_{\epsilon}}} I_{\rho_{\epsilon}}(u)
$$

and there is $\beta>0$ independent of $\epsilon$, such that

$$
\beta \leq\|u\|_{\rho_{\epsilon}}^{2}, \quad \forall u \in X^{\epsilon}
$$

and so,

$$
\beta \leq C_{\rho_{\epsilon}}, \quad \forall \epsilon>0 .
$$

From (3.2), if $C_{\rho_{\epsilon}}$ is a critical value of $I_{\rho_{\epsilon}}$ then it is the least energy critical value of $I_{\rho_{\epsilon}}$. Hereafter, we say that $C_{\rho_{\epsilon}}$ is the ground state level of $I_{\rho_{\epsilon}}$.

Now, we consider the following equation

$$
(-\Delta)^{\alpha} u+Q(\xi) u=K(\xi)|u|^{p-1} u, \quad x \in \mathbb{R}^{n},
$$

where $\xi \in \mathbb{R}^{n}$ is regard as a parameter instead of an independent variable. We define the energy functional $J_{\xi}: H^{\alpha}\left(\mathbb{R}^{n}\right) \rightarrow \mathbb{R}$ associated with (3.5) by

$$
\begin{aligned}
J_{\xi}(u)= & \frac{1}{2}\left(\int_{\mathbb{R}^{n}} \int_{\mathbb{R}^{n}} \frac{|u(x+z)-u(x)|^{2}}{|z|^{n+2 \alpha}} d z d x+\int_{\mathbb{R}^{n}} Q(\xi)|u(x)|^{2} d x\right) \\
& -\frac{1}{p+1} \int_{\mathbb{R}^{n}} K(\xi)|u(x)|^{p+1} d x .
\end{aligned}
$$

Let

$$
C(\xi)=\inf _{u \in \mathcal{N}_{\xi}} J_{\xi}(u)
$$

the ground state energy associated with (3.5), where $\mathcal{N}_{\xi}$ is the Nehari manifold defined as

$$
\mathcal{N}_{\xi}=\left\{u \in H^{\alpha}\left(\mathbb{R}^{n}\right) \backslash\{0\}: J_{\xi}^{\prime}(u) u=0\right\} .
$$

Arguing as above, we see that $C(\xi)>0$ and

$$
C(\xi)=\inf _{v \in H^{\alpha}\left(\mathbb{R}^{n}\right) \backslash\{0\}} \max _{t>0} J_{\xi}(t v)=\inf _{\gamma \in \Gamma_{\xi}} \max _{t \in[0,1]} J_{\xi}(\gamma(t)),
$$

where

$$
\Gamma_{\xi}=\left\{\gamma \in C\left([0,1], H^{\alpha}\left(\mathbb{R}^{n}\right)\right): \quad \gamma(0)=0, \quad J_{\xi}(\gamma(1))<0\right\} .
$$

By [8], we know that for each $\xi \in \mathbb{R}^{n}$, problem (3.5) has a nontrivial nonnegative ground state solution. Thus, $C(\xi)$ is the least critical value of $J_{\xi}$. Next, we will study the continuity of $C(\xi)$.

Lemma 3.1. The function $\xi \rightarrow C(\xi)$ is continuous. 
Proof. Set $\left\{\xi_{r}\right\} \subset \mathbb{R}^{n}$ and $\xi_{0} \in \mathbb{R}^{n}$ with

$$
\xi_{r} \rightarrow \xi_{0} \quad \text { in } \mathbb{R}^{n} \text {. }
$$

By using the conditions on $\rho, Q$ and $K$, we know that

$$
\liminf _{\xi \in \mathbb{R}^{n}} C(\xi)>0 \text { and } \quad \limsup _{\xi \in \mathbb{R}^{n}} C(\xi)<+\infty .
$$

Next, we denote by $v_{r} \in H^{\alpha}\left(\mathbb{R}^{n}\right)$ the function which satisfies

$$
J_{\xi_{r}}\left(v_{r}\right)=C\left(\xi_{r}\right) \quad \text { and } \quad J_{\xi_{r}}^{\prime}\left(v_{r}\right)=0
$$

In the sequel, we will consider two sequences $\left\{\xi_{r_{j}}\right\}$ and $\left\{\xi_{r_{k}}\right\}$ such that

$$
C\left(\xi_{r_{j}}\right) \geq C\left(\xi_{0}\right) \quad \forall r_{j}
$$

and

$$
C\left(\xi_{r_{k}}\right) \leq C\left(\xi_{0}\right) \quad \forall r_{k} .
$$

Analysis of $(I)$ : From the above commentaries, we know that $\left.\left\{C_{(} \xi_{r_{j}}\right)\right\}$ is bounded. Therefore, there are a subsequence $\left\{\xi_{r_{j_{i}}}\right\} \subset\left\{\xi_{r_{j}}\right\}$ and $C_{0}>0$ such that

$$
C\left(\xi_{r_{j}}\right) \rightarrow C_{0}
$$

In the sequel, we will use the following notations:

$$
v_{i}=v_{r_{j_{i}}} \text { and } \quad \xi_{i}=\xi_{r_{j_{i}}} .
$$

Thereby,

$$
\xi_{i} \rightarrow \xi_{0} \quad \text { and } \quad C\left(\xi_{i}\right) \rightarrow C_{0}
$$

Claim A: $C_{0}=C\left(\xi_{0}\right)$. From (I),

$$
\lim _{i} C\left(\xi_{i}\right) \geq C\left(\xi_{0}\right)
$$

and so,

$$
C_{0} \geq C\left(\xi_{0}\right)
$$

In the sequel, we set $w_{0} \in H^{\alpha}\left(\mathbb{R}^{n}\right)$ be a function satisfying

$$
J_{\xi_{0}}\left(w_{0}\right)=C\left(\xi_{0}\right) \quad \text { and } \quad J_{\xi_{0}}^{\prime}\left(w_{0}\right)=0 .
$$

Moreover, we denote by $t_{i}>0$ the real number which verifies

$$
J_{\xi_{i}}\left(t_{i} w_{0}\right)=\max _{t \geq 0} J_{\xi_{i}}\left(t_{i} w_{0}\right) .
$$

Thus, by definition of $C\left(\xi_{0}\right)$,

$$
C\left(\xi_{i}\right) \leq J_{\xi_{i}}\left(t_{i} w_{0}\right) .
$$

It is possible to prove that $\left\{t_{i}\right\}$ is a bounded sequence, then without lost of generality we can assume that $t_{i} \rightarrow t_{0}$. Now, by using the fact that the functions $\rho$ and $K$ are continuous, the Lebesgue's Theorem gives

$$
\lim _{i} J_{\xi_{i}}\left(t_{i} w_{0}\right)=J_{\xi_{0}}\left(t_{0} w_{0}\right) \leq J_{\xi_{0}}\left(w_{0}\right)=C\left(\xi_{0}\right),
$$

leading to

$$
C_{0} \leq C\left(\xi_{0}\right)
$$


From (3.7)- (3.8),

$$
C\left(\xi_{0}\right)=C_{0}
$$

The above study implies that

$$
\lim _{i} C\left(\xi_{r_{j_{i}}}\right)=C\left(\xi_{0}\right) \text {. }
$$

Analysis of $(I I):$ By using the definition of $\left\{v_{r}\right\}$, it is easy to prove that $\left\{v_{r}\right\}$ is a bounded sequence in $H^{\alpha}\left(\mathbb{R}^{n}\right)$. Consequently, there is $v_{0} \in H^{\alpha}\left(\mathbb{R}^{n}\right)$ such that

$$
v_{r} \rightarrow v_{0} \quad \text { in } \quad H^{\alpha}\left(\mathbb{R}^{n}\right) .
$$

By using Lemma 2.1, we can assume that $v_{0} \neq 0$, because for any translation of the type $\tilde{v}_{n}(x)=v_{n}\left(x+y_{n}\right)$ also satisfies

$$
J_{\xi_{r}}\left(\tilde{v}_{r}\right)=C\left(\xi_{r}\right) \quad \text { and } \quad J_{\xi_{r}}^{\prime}\left(\tilde{v}_{r}\right)=0
$$

The above information permits to conclude that $v_{0}$ is a nontrivial solution of the problem

$$
(-\Delta)^{\alpha} u+Q\left(\xi_{0}\right) u=K\left(\xi_{0}\right)|u|^{p-1} u \text { in } \mathbb{R}^{n}, \quad u \in H^{\alpha}\left(\mathbb{R}^{n}\right) .
$$

By Fatous' lemma, it is possible to prove that

$$
\liminf _{r} J_{\xi_{r}}\left(v_{r}\right) \geq J_{\xi_{0}}\left(v_{0}\right) \text {. }
$$

On the other hand, there is $s_{r}>0$ such that

$$
C\left(\xi_{r}\right) \leq J_{\xi_{r}}\left(s_{r} v_{0}\right) \quad \forall r .
$$

So

$$
\limsup _{r} J_{\xi_{r}}\left(v_{r}\right)=\limsup _{r} C\left(\xi_{r}\right) \leq \limsup _{r} J_{\xi_{r}}\left(s_{r} v_{0}\right)=J_{\xi_{0}}\left(v_{0}\right) .
$$

From (3.10)-(3.11),

$$
\lim _{r} J_{\xi_{n}}\left(v_{n}\right)=J_{\xi_{0}}\left(v_{0}\right)
$$

The last limit yields

$$
v_{r} \rightarrow v_{0} \quad \text { in } \quad H^{\alpha}\left(\mathbb{R}^{n}\right) .
$$

Since $\left\{C\left(\xi_{r_{j_{k}}}\right)\right\}$ is bounded, there are a subsequence $\left\{\xi_{r_{j_{k}}}\right\} \subset\left\{\xi_{r_{j}}\right\}$ and $C_{*}>0$ such that

$$
C\left(\xi_{r_{k}}\right) \rightarrow C_{*}
$$

In the sequel, we will use the following notations:

$$
v_{k}=v_{r_{j_{k}}} \text { and } \quad \xi_{k}=\xi_{r_{j_{k}}} .
$$

Thus,

$$
v_{k} \rightarrow v_{0}, \quad \xi_{k} \rightarrow \xi_{0} \quad \text { and } \quad C\left(\xi_{k}\right) \rightarrow C_{*} .
$$

In what follows, we denote by $t_{k}>0$ the real number which verifies

$$
J_{\xi_{0}}\left(t_{k} v_{k}\right)=\max _{t \geq 0} J_{\xi_{0}}\left(t v_{k}\right)
$$

Thus, by definition of $C\left(\xi_{0}\right)$,

$$
C\left(\xi_{0}\right) \leq J_{\xi_{0}}\left(t_{k} v_{k}\right)
$$


It is possible to prove that $\left\{t_{k}\right\}$ is a bounded sequence, then without lost of generality we can assume that $t_{k} \rightarrow t_{*}$. Now, by using the fact that the functions $\rho$ and $K$ are continuous, the Lebesgue's Theorem gives

$$
\lim _{k} J_{\xi_{0}}\left(t_{k} v_{k}\right)=J_{\xi_{0}}\left(t_{*} v_{0}\right)=\lim _{k} J_{\xi_{k}}\left(t_{k} v_{k}\right) \leq \lim _{k} C\left(\xi_{k}\right)=C_{*} .
$$

Thereby,

$$
C\left(\xi_{0}\right) \leq C_{*}
$$

On the other hand, from $(I I)$,

$$
\lim _{k} C\left(\xi_{k}\right) \leq C\left(\xi_{0}\right)
$$

leading to

$$
C_{*} \geq C\left(\xi_{0}\right)
$$

From (3.12)-(3.13),

$$
C_{*}=C\left(\xi_{0}\right) .
$$

The above study implies that

$$
\lim _{k} C\left(\xi_{n_{j_{k}}}\right)=C\left(\xi_{0}\right)
$$

From $(I)$ and $(I I)$,

$$
\lim _{r}\left(\xi_{r}\right)=C\left(\xi_{0}\right)
$$

showing the lemma.

In what follows, we denote by $D$ the ground state level of the function $J: H^{\alpha}\left(\mathbb{R}^{n}\right) \rightarrow \mathbb{R}$ given by

$$
J(u)=\frac{1}{2}\left(\int_{\mathbb{R}^{n}} \int_{\mathbb{R}^{n}} \frac{|u(x)-u(z)|^{2}}{|x-z|^{n+2 \alpha}} d z d x+\int_{\mathbb{R}^{n}}|u(x)|^{2} d x\right)-\frac{1}{p+1} \int_{\mathbb{R}^{n}}|u|^{p+1} d x
$$

Using the above notations, we have the following lemma

Lemma 3.2. The functions $C(\xi)$ verifies the following relation

$$
C(\xi)=\frac{Q(\xi)^{\frac{p+1}{p-1}-\frac{n}{2 \alpha}}}{K(\xi)^{\frac{2}{p-1}}} D, \quad \forall \xi \in \mathbb{R}^{n} .
$$

Proof. Let $u \in H^{\alpha}\left(\mathbb{R}^{n}\right)$ be a function verifying

$$
J(u)=D \quad \text { and } \quad J^{\prime}(u)=0 .
$$

For each $\xi \in \mathbb{R}^{n}$ fixed, let $\sigma^{2 \alpha}=\frac{1}{Q(\xi)}$ and define

$$
w(x)=\left[\frac{Q(\xi)}{K(\xi)}\right]^{\frac{1}{p-1}} u\left(\frac{x}{\sigma}\right) .
$$


Then, doing the change of variable $x=\sigma \tilde{x}$ and $z=\sigma \tilde{z}$ we obtain

$$
\begin{aligned}
& J_{\xi}(w)=\frac{1}{2}\left(\int_{\mathbb{R}^{n}} \int_{\mathbb{R}^{n}} \frac{|w(x+z)-w(x)|^{2}}{|z|^{n+2 \alpha}} d z d x+\int_{\mathbb{R}^{n}} Q(\xi) w^{2} d x\right)-\frac{1}{p+1} \int_{\mathbb{R}^{n}} K(\xi)|w|^{p+1} d x \\
& =\frac{Q(\xi)}{2}\left(\sigma^{2 \alpha} \int_{\mathbb{R}^{n}} \int_{\mathbb{R}^{n}} \frac{|w(x+z)-w(x)|^{2}}{|z|^{n+2 \alpha}} d z d x+\int_{\mathbb{R}^{n}} w^{2}(x) d x\right)-\frac{1}{p+1} \int_{\mathbb{R}^{n}} K(\xi)|w|^{p+1} d x \\
& =\frac{Q(\xi)^{\frac{p+1}{p-1}}}{K(\xi)^{\frac{2}{p-1}}}\left[\left(\frac{\sigma^{2 \alpha}}{2} \int_{\mathbb{R}^{n}} \int_{\mathbb{R}^{n}} \frac{\left|u\left(\frac{x}{\sigma}+\frac{z}{\sigma}\right)-u\left(\frac{x}{\sigma}\right)\right|^{2}}{|z|^{n+2 \alpha}} d z d x+\frac{1}{2} \int_{\mathbb{R}^{n}}\left|u\left(\frac{x}{\sigma}\right)\right|^{2} d x\right)-\frac{1}{p+1} \int_{\mathbb{R}^{n}}\left|u\left(\frac{x}{\sigma}\right)\right|^{p+1} d x\right] \\
& =\frac{Q(\xi)^{\frac{p+1}{p-1}-\frac{n}{2 \alpha}}}{K(\xi)^{\frac{2}{p-1}}} J(u) .
\end{aligned}
$$

A similar argument also gives $J_{\xi}^{\prime}(w)(w)=0$, from where it follows

$$
C(\xi) \leq \frac{Q(\xi)^{\frac{p+1}{p-1}-\frac{n}{2 \alpha}}}{K(\xi)^{\frac{2}{p-1}}} D, \quad \forall \xi \in \mathbb{R}^{n} .
$$

The reverse inequality is obtained of the same way, finishing the proof.

As a byproduct of the last proof, we have the following corollary

Corollary 3.1. By Lemma 3.2, if there is $\xi_{0} \in \mathbb{R}^{n}$ such that

$$
\frac{Q\left(\xi_{0}\right)^{\frac{p+1}{p-1}-\frac{n}{2 \alpha}}}{K\left(\xi_{0}\right)^{\frac{2}{p-1}}}<\frac{Q_{\infty}^{\frac{p+1}{p-1}-\frac{n}{2 \alpha}}}{K_{\infty}^{\frac{2}{p-1}}},
$$

we have

$$
\inf _{\xi \in \mathbb{R}^{n}} C(\xi)<\liminf _{|\xi| \rightarrow+\infty} C(\xi)=C(\infty),
$$

where $C(\infty)$ is the mountain pass level of the functionals $J_{\infty}: H^{\alpha}\left(\mathbb{R}^{n}\right) \rightarrow \mathbb{R}$ given by

$J_{\infty}(u)=\frac{1}{2}\left(\int_{\mathbb{R}^{n}} \int_{\mathbb{R}^{n}} \frac{|u(x)-u(z)|^{2}}{|x-z|^{n+2 \alpha}} d z d x+\int_{\mathbb{R}^{n}} Q_{\infty}|u|^{2} d x\right)-\frac{1}{p+1} \int_{\mathbb{R}^{n}} K_{\infty}|u|^{p+1} d x$.

The next lemma studies the behavior of function $C_{\rho_{\epsilon}}(\xi)$ when $\epsilon$ goes to 0 .

Lemma 3.3. $\limsup _{\epsilon \rightarrow 0} C_{\rho_{\epsilon}} \leq \inf _{\xi \in \mathbb{R}^{n}} C(\xi)$. Hence, $\limsup _{\epsilon \rightarrow 0} C_{\rho_{\epsilon}}<C(\infty)$.

Proof. Fix $\xi_{0} \in \mathbb{R}^{N}$ and $w \in H^{\alpha}\left(\mathbb{R}^{n}\right)$ with

$$
J_{\xi_{0}}(w)=\max _{t \geq 0} J_{\xi_{0}}(t w)=C\left(\xi_{0}\right) \quad \text { and } \quad J_{\xi_{0}}^{\prime}(w)=0
$$

where

$$
J_{\xi_{0}}(u)=\frac{1}{2}\left(\int_{\mathbb{R}^{n}} \int_{\mathbb{R}^{n}} \frac{|u(x)-u(z)|^{2}}{|x-z|^{n+2 \alpha}} d z d x+\int_{\mathbb{R}^{n}} Q\left(\xi_{0}\right)|u(x)|^{2} d x\right)-\frac{1}{p+1} \int_{\mathbb{R}^{n}} K\left(\xi_{0}\right)|u|^{p+1} d x .
$$


Then, we take $w_{\epsilon}(x)=w\left(x-\frac{\xi_{0}}{\epsilon}\right)$ and $t_{\epsilon}>0$ satisfying

$$
C_{\rho_{\epsilon}} \leq I_{\rho_{\epsilon}}\left(t_{\epsilon} w_{\epsilon}\right)=\max _{t \geq 0} I_{\rho_{\epsilon}}\left(t w_{\epsilon}\right) .
$$

The change of variable $\tilde{x}=x-\frac{\xi_{0}}{\epsilon}$ gives

$$
\begin{aligned}
I_{\rho_{\epsilon}}\left(t_{\epsilon} w_{\epsilon}\right) & =\frac{t_{\epsilon}^{2}}{2}\left(\int_{\mathbb{R}^{n}} \int_{B\left(0, \frac{1}{\epsilon} \rho(\epsilon x)\right)} \frac{\left|w_{\epsilon}(x+z)-w_{\epsilon}(x)\right|^{2}}{|z|^{n+2 \alpha}} d x d x+\int_{\mathbb{R}^{n}} Q(\epsilon x) w_{\epsilon}^{2}(x) d x\right) \\
& -\frac{t_{\epsilon}^{p+1}}{p+1} \int_{\mathbb{R}^{n}} K(\epsilon x) w_{\epsilon}^{p+1}(x) d x \\
& =\frac{t_{\epsilon}^{2}}{2}\left(\int_{\mathbb{R}^{n}} \int_{B\left(0, \frac{1}{\epsilon} \rho\left(\epsilon \tilde{x}+\xi_{0}\right)\right)} \frac{|w(\tilde{x}+z)-w(\tilde{x})|^{2}}{|z|^{n+2 \alpha}} d z d \tilde{x}+\int_{\mathbb{R}^{n}} Q\left(\epsilon \tilde{x}+\xi_{0}\right) w^{2}(\tilde{x}) d \tilde{x}\right) \\
& -\frac{t_{\epsilon}^{p+1}}{p+1} \int_{\mathbb{R}^{n}} K\left(\epsilon \tilde{x}+\xi_{0}\right) w^{p+1}(\tilde{x}) d \tilde{x} .
\end{aligned}
$$

Thereby, considering a sequence $\epsilon_{n} \rightarrow 0$, the fact that $I_{\rho_{\epsilon_{n}}}^{\prime}\left(t_{\epsilon_{n}} w_{\epsilon_{n}}\right)\left(t_{\epsilon_{n}} w_{\epsilon_{n}}\right)=$ 0 yields $\left\{t_{\epsilon_{n}}\right\}$ is bounded. Thus, we can assume that

$$
t_{\epsilon_{n}} \rightarrow t_{*}>0
$$

for some $t_{*}>0$. Using a change variable as above, we can infer that

$$
J_{\xi_{0}}^{\prime}\left(t_{*} w\right)\left(t_{*} w\right)=0 .
$$

On the other hand, we know that $J_{\xi_{0}}^{\prime}(w)(w)=0$. Then by uniqueness, we must have

$$
t_{*}=1
$$

From this,

$$
I_{\rho_{\epsilon_{n}}}\left(t_{\epsilon_{n}} w_{\epsilon_{n}}\right) \rightarrow J_{\xi_{0}}(w)=C\left(\xi_{0}\right) \text { as } \epsilon \rightarrow 0 .
$$

As the point $\xi_{0} \in \mathbb{R}^{n}$ is arbitrary, the lemma is proved.

Theorem 3.1. For $\epsilon>0$ small enough, the problem $\left(P^{\prime}\right)$ has a positive least energy solution.

Proof. In what follows, we denote by $\left\{u_{k}\right\} \subset H^{\alpha}\left(\mathbb{R}^{N}\right)$ a sequence satisfying

$$
I_{\rho_{\epsilon}}\left(u_{k}\right) \rightarrow C_{\rho_{\epsilon}} \text { and } I_{\rho_{\epsilon}}^{\prime}\left(u_{k}\right) \rightarrow 0 .
$$

If $u_{k} \rightarrow 0$ in $H^{\alpha}\left(\mathbb{R}^{N}\right)$, then

$$
u_{k} \rightarrow 0 \text { in } L_{l o c}^{p}\left(\mathbb{R}^{n}\right) \text { for } p \in\left[2,2_{\alpha}^{*}\right) .
$$

By $\left(H_{0}\right)$, we can take $\delta, R>0$ such that

$$
Q_{\infty}-\delta \leq Q(x) \leq Q_{\infty}+\delta \text { and } K_{\infty}-\delta \leq K(x) \leq K_{\infty}+\delta
$$


for all $|x| \geq R$. Then, for all $t \geq 0$,

$$
\begin{aligned}
I_{\rho_{\epsilon}}\left(t u_{k}\right) & =I_{\epsilon, \infty}^{\delta}\left(t u_{k}\right)+\frac{t^{2}}{2} \int_{\mathbb{R}^{n}}\left[Q(x)-Q_{\infty}+\delta\right]\left|u_{k}(x)\right|^{2} d x \\
& +\frac{t^{p+1}}{p+1} \int_{\mathbb{R}^{n}}\left[K_{\infty}+\delta-K(x)\right]\left|u_{k}(x)\right|^{p+1} d x \\
& \geq I_{\epsilon, \infty}^{\delta}\left(t u_{k}\right)+\frac{t^{2}}{2} \int_{B\left(0, \frac{R}{\epsilon}\right)}\left[Q(x)-Q_{\infty}+\delta\right]\left|u_{k}(x)\right|^{2} d x \\
& +\frac{t^{p+1}}{p+1} \int_{B\left(0, \frac{R}{\epsilon}\right)}\left[K_{\infty}+\delta-K(x)\right]\left|u_{k}(x)\right|^{p+1} d x
\end{aligned}
$$

where

$$
\begin{aligned}
I_{\epsilon, \infty}^{\delta}(u) & =\frac{1}{2}\left(\int_{\mathbb{R}^{n}} \int_{B\left(0, \frac{1}{\epsilon} \rho(\epsilon x)\right.} \frac{|u(x+z)-u(x)|^{2}}{|z|^{n+2 \alpha}} d x d x+\int_{\mathbb{R}^{n}}\left(Q_{\infty}-\delta\right)|u(x)|^{2} d x\right) \\
& -\frac{1}{p+1} \int_{\mathbb{R}^{n}}\left(K_{\infty}+\delta\right)|u(x)|^{p+1} d x .
\end{aligned}
$$

Now we know that there exists a bounded sequence $\left\{\tau_{k}\right\}$ such that

$$
I_{\epsilon, \infty}^{\delta}\left(\tau_{k} u_{k}\right) \geq C\left(\frac{\rho(\epsilon x)}{\epsilon}, Q_{\infty}-\delta, K_{\infty}+\delta\right)
$$

where

$$
C\left(\frac{\rho(\epsilon x)}{\epsilon}, Q_{\infty}-\delta, K_{\infty}+\delta\right)=\inf _{v \in H^{\alpha}(\mathbb{R}) \backslash\{0\}} \sup _{t \geq 0} I_{\epsilon, \infty}^{\delta}(t v)
$$

Thus,

$$
\begin{aligned}
C_{\rho_{\epsilon}} & \geq C\left(\frac{\rho(\epsilon x)}{\epsilon}, Q_{\infty}-\delta, K_{\infty}+\delta\right)+\frac{\tau_{k}^{2}}{2} \int_{B\left(0, \frac{R}{\epsilon}\right)}\left[Q(x)-Q_{\infty}+\delta\right]\left|u_{k}(x)\right|^{2} d x \\
& +\frac{\tau_{k}^{p+1}}{p+1} \int_{B\left(0, \frac{R}{\epsilon}\right)}\left[K_{\infty}+\delta-K(x)\right]\left|u_{k}(x)\right|^{p+1} d x
\end{aligned}
$$

Taking the limit as $k \rightarrow \infty$, and after $\delta \rightarrow 0$, we find

$$
c_{\rho_{\epsilon}} \geq C\left(\frac{\rho(\epsilon x)}{\epsilon}, Q_{\infty}, K_{\infty}\right)
$$

where $C\left(\frac{\rho(\epsilon x)}{\epsilon}, Q_{\infty}, K_{\infty}\right)$ denotes the mountain pass level of the functional

$$
\begin{aligned}
I_{\infty, \xi}^{0}(u)= & \frac{1}{2}\left(\int_{\mathbb{R}^{n}} \int_{B\left(0, \frac{1}{\epsilon} \rho(\epsilon x)\right.} \frac{|u(x+z)-u(x)|^{2}}{|z|^{n+2 \alpha}} d z d x+\int_{\mathbb{R}^{n}} Q_{\infty}|u|^{2} d x\right)- \\
& \frac{1}{p+1} \int_{\mathbb{R}^{n}} K_{\infty} u^{p+1} d x .
\end{aligned}
$$

A standard argument shows that

$$
\liminf _{\epsilon \rightarrow 0} C\left(\frac{\rho(\epsilon x)}{\epsilon}, Q_{\infty}, K_{\infty}\right) \geq C(\infty)
$$


Therefore, if there is $\epsilon_{n} \rightarrow 0$ such that the $(P S)_{C_{\rho_{\epsilon_{n}}}}$ sequence has weak limit equal to zero, we must have

$$
C_{\rho_{\epsilon_{n}}} \geq C\left(\frac{\rho\left(\epsilon_{n} x\right)}{\epsilon_{n}}, Q_{\infty}, K_{\infty}\right), \quad \forall n \in \mathbb{N},
$$

leading to

$$
\liminf _{n \rightarrow+\infty} C_{\rho_{\epsilon_{n}}} \geq C(\infty),
$$

which contradicts Lemma 3.3. This proves that the weak limit is non trivial for $\epsilon>0$ small enough and standard arguments show that its energy is equal to $C_{\rho_{\epsilon}}$, showing the desired result.

\section{Concentration of the solutions $u_{\epsilon}$}

Lemma 4.1. If $v_{\epsilon}$ is family solutions of $\left(P^{\prime}\right)$ with critical value $C_{\rho_{\epsilon}}$, then there exists a family $\left\{y_{\epsilon}\right\}$ and positive constants $R$ and $\beta$ such that

$$
\liminf _{\epsilon \rightarrow 0^{+}} \int_{B\left(y_{\epsilon}, R\right)}\left|v_{\epsilon}\right|^{2} d x \geq \beta>0 .
$$

Proof. First we note that, by $\left(H_{1}\right)$ and $\left(H_{3}\right)$ we have

$$
\begin{aligned}
I_{\rho_{\epsilon}}(v) \geq I_{*}(v) & =\frac{1}{2}\left(\int_{\mathbb{R}^{n}} \int_{B\left(0, \rho_{0}\right)} \frac{|v(x+z)-v(x)|^{2}}{|z|^{n+2 \alpha}} d z d x+\int_{\mathbb{R}^{n}} a_{1}|v|^{2} d x\right) \\
& -\frac{1}{p+1} \int_{\mathbb{R}^{n}} a_{2}|u|^{p+1} d x .
\end{aligned}
$$

Let $\mathcal{N}_{*}=\left\{v \in H^{\alpha}\left(\mathbb{R}^{n}\right) \backslash\{0\}: \quad I_{*}^{\prime}(v) v=0\right\}$. Then, for each $v \in \mathcal{N}_{*}$ there exists unique $t_{v}>0$ such that $t_{v} v \in \mathcal{N}_{*}$. Hence,

$$
\begin{aligned}
0<C\left(\rho_{0}, a_{1}, a_{2}\right) & =\inf _{v \in \mathcal{N}_{*}} I_{*}(v) \leq \inf _{v \in \mathcal{N}_{*}} I_{\rho_{\epsilon}}(v) \\
& \leq \inf _{v \in \mathcal{N}_{*}} I_{\rho_{\epsilon}}\left(t_{v} v\right)=\inf _{u \in \mathcal{N}_{\rho_{\epsilon}}} I_{\rho_{\epsilon}}(u)=C_{\rho_{\epsilon}} .
\end{aligned}
$$

Now, by contradiction, if (4.1) does not hold, then there exists a sequence $v_{k}=v_{\epsilon_{k}}$ such that

$$
\lim _{k \rightarrow \infty} \sup _{y \in \mathbb{R}^{n}} \int_{B(y, R)}\left|v_{k}\right|^{2} d x=0 .
$$

By Lemma 2.1, $v_{k} \rightarrow 0$ in $L^{q}\left(\mathbb{R}^{n}\right)$ for any $2<q<2_{\alpha}^{*}$. However, this is impossible since by (4.2)

$$
\begin{aligned}
0<C\left(\rho_{0}, a_{1}, a_{2}\right) \leq C_{\rho_{\epsilon}} & =I_{\rho_{\epsilon}}\left(v_{\epsilon}\right)-\frac{1}{2} I_{\rho_{\epsilon}}^{\prime}\left(v_{\epsilon}\right) v_{\epsilon} \\
& =\frac{p-1}{2(p+1)} \int_{\mathbb{R}^{n}} K(\epsilon x)\left|v_{\epsilon}\right|^{p+1} d x \\
& \leq \frac{p-1}{2(p+1)} \int_{\mathbb{R}^{n}} a_{2}\left|v_{\epsilon}\right|^{p+1} d x \rightarrow 0, \text { as } k \rightarrow \infty .
\end{aligned}
$$


Now let

$$
w_{\epsilon}(x)=v_{\epsilon}\left(x+y_{\epsilon}\right)=u_{\epsilon}\left(\epsilon x+\epsilon y_{\epsilon}\right) \text {, }
$$

then by (4.5),

$$
\liminf _{\epsilon \rightarrow 0^{+}} \int_{B(0, R)}\left|w_{\epsilon}\right|^{2} d x \geq \beta>0 .
$$

To continue, we consider the rescaled scope function $\bar{\rho}_{\epsilon}$, defined as,

$$
\bar{\rho}_{\epsilon}(x)=\frac{1}{\epsilon} \rho\left(\epsilon x+\epsilon y_{\epsilon}\right)
$$

and then $w_{\epsilon}$ satisfies the equation

$$
(-\Delta) \bar{\rho}_{\epsilon} w_{\epsilon}(x)+Q\left(\epsilon x+\epsilon y_{\epsilon}\right) w_{\epsilon}(x)=K\left(\epsilon x+\epsilon y_{\epsilon}\right)\left|w_{\epsilon}(x)\right|^{p-1} w_{\epsilon}(x), \text { in } \mathbb{R}^{n} \text {. }
$$

Lemma 4.2. The sequence $\left\{\epsilon y_{\epsilon}\right\}$ is bounded. Moreover, if $\epsilon_{m} y_{\epsilon_{m}} \rightarrow \xi^{*}$, then

$$
C\left(\xi^{*}\right)=\inf _{\xi \in \mathbb{R}^{n}} C(\xi) .
$$

Proof. Suppose by contradiction that $\left|\epsilon_{m} y_{\epsilon_{m}}\right| \rightarrow \infty$ and consider the function $w_{\epsilon_{m}}$ defined by (4.3), which satisfies (4.5). Since $\left\{C_{\rho_{\epsilon_{m}}}\right\}$ is bounded, so the sequence $\left\{w_{m}\right\}$ is also bounded in $H^{\alpha}\left(\mathbb{R}^{n}\right)$. Then $w_{m} \rightarrow w$ in $H^{\alpha}\left(\mathbb{R}^{n}\right)$, and $w \neq 0$ by Lemma 4.1. Now, by (4.5) we get the following equality

$$
\begin{aligned}
& \int_{\mathbb{R}^{n}} \int_{B\left(0, \frac{1}{\epsilon_{m}} \rho\left(\epsilon_{m} x+\epsilon_{m} y_{\epsilon_{m}}\right)\right)} \frac{\left[w_{m}(x+z)-w_{m}(x)\right][w(x+z)-w(x)]}{|z|^{n+2 \alpha}} d z d x \\
& +\int_{\mathbb{R}^{n}} Q\left(\epsilon_{m} x+\epsilon_{m} y_{\epsilon_{m}}\right) w_{m} w d x=\int_{\mathbb{R}^{n}} K\left(\epsilon_{m} x+\epsilon_{m} y_{\epsilon_{m}}\right)\left|w_{m}\right|^{p-1} w_{m} w d x .
\end{aligned}
$$

So, by Fatou's Lemma we get

$$
\int_{\mathbb{R}^{n}} \int_{\mathbb{R}^{n}} \frac{|w(x+z)-w(x)|^{2}}{|z|^{n+2 \alpha}} d z d x+\int_{\mathbb{R}^{n}} Q_{\infty}|w|^{2} d x \leq \int_{\mathbb{R}^{n}} K_{\infty}|w|^{p+1} d x
$$

Let $\theta>0$ such that

$$
J_{\infty}(\theta w)=\max _{t \geq 0} J_{\infty}(t w)
$$

From (4.6),,$\theta \in(0,1]$, whence

$$
\begin{aligned}
C(\infty) & \leq J_{\infty}(\theta w)-\frac{1}{2} J_{\infty}^{\prime}(\theta w) \theta w=\left(\frac{1}{2}-\frac{1}{p+1}\right) \theta^{p+1} \int_{\mathbb{R}^{n}} K_{\infty}|w(x)|^{p+1} d x \\
& \leq\left(\frac{1}{2}-\frac{1}{p+1}\right) \int_{\mathbb{R}^{n}} K_{\infty}|w(x)|^{p+1} d x \\
& \leq\left(\frac{1}{2}-\frac{1}{p+1}\right) \liminf _{m \rightarrow \infty} \int_{\mathbb{R}^{n}} K\left(\epsilon_{m} x+\epsilon_{m} y_{\epsilon_{m}}\right)\left|w_{m}(x)\right|^{p+1} d x \\
& =\liminf _{m \rightarrow \infty} C_{\rho_{\epsilon_{n}}}<C(\infty)
\end{aligned}
$$

which is a contradiction. So $\left\{\epsilon y_{\epsilon}\right\}$ is bounded. Thus, there exists a subsequence of $\left\{\epsilon y_{\epsilon}\right\}$ such that $\epsilon_{m} y_{\epsilon_{m}} \rightarrow \xi^{*}$. 
Repeating above arguments, define the function

$$
w_{m}(x)=v_{\epsilon_{m}}\left(x+y_{\epsilon_{m}}\right)=u_{\epsilon_{m}}\left(\epsilon_{m} x+\epsilon_{m} y_{\epsilon_{m}}\right) .
$$

This function satisfies the equation (4.5), and again $\left\{w_{m}\right\}$ is bounded in $H^{\alpha}\left(\mathbb{R}^{n}\right)$. Then $w_{m} \rightarrow w$ in $H^{\alpha}\left(\mathbb{R}^{n}\right)$, where $w$ satisfy the following equation

$$
(-\Delta)^{\alpha} w+Q\left(\xi^{*}\right) w=K\left(\xi^{*}\right)|w|^{p-1} w, \quad x \in \mathbb{R}^{n},
$$

in the weak sense. Furthermore, associated to (4.7) we have the energy functional

$$
\begin{aligned}
J_{\xi^{*}}(u) & =\frac{1}{2}\left(\int_{\mathbb{R}^{n}} \int_{\mathbb{R}^{n}} \frac{|u(x+z)-u(x)|^{2}}{|z|^{n+2 \alpha}} d z d x+\int_{\mathbb{R}^{n}} Q\left(\xi^{*}\right)|u(x)|^{2} d x\right) \\
& -\frac{1}{p+1} \int_{\mathbb{R}^{n}} K\left(\xi^{*}\right)|u(x)|^{p+1} d x .
\end{aligned}
$$

Using $w$ as a test function in (4.5) and taking the limit of $m \rightarrow+\infty$, we get

$$
\int_{\mathbb{R}^{n}} \int_{\mathbb{R}^{n}} \frac{|w(x+z)-w(x)|^{2}}{|z|^{n+2 \alpha}} d x d x+\int_{\mathbb{R}^{n}} Q\left(\xi^{*}\right)|w(x)|^{2} d x \leq \int_{\mathbb{R}^{n}} K\left(\xi^{*}\right)|w|^{p+1} d x,
$$

which implies that there exists $\theta \in(0,1]$ such that

$$
J_{\xi^{*}}(\theta w)=\max _{t \geq 0} J_{\xi^{*}}(t w)
$$

So, by Lemma 3.3 ,

$$
\begin{aligned}
C\left(\xi^{*}\right) & \leq J_{\xi^{*}}(\theta w)=\left(\frac{1}{2}-\frac{1}{p+1}\right) \theta^{p+1} \int_{\mathbb{R}^{n}} K\left(\xi^{*}\right)|w(x)|^{p+1} d x \\
& \leq\left(\frac{1}{2}-\frac{1}{p+1}\right) \liminf _{m \rightarrow \infty} \int_{\mathbb{R}^{n}} K\left(\epsilon_{m} x+\epsilon_{m} y_{\epsilon_{m}}\right)\left|w_{m}(x)\right|^{p+1} d x \\
& =\liminf _{m \rightarrow \infty}\left[I_{\rho_{\epsilon_{m}}}\left(v_{\epsilon_{m}}\right)-I_{\rho_{\epsilon_{m}}}^{\prime}\left(v_{\epsilon_{m}}\right) v_{\epsilon_{m}}\right] \\
& =\liminf _{m \rightarrow \infty} C_{\rho_{\epsilon_{m}}} \leq \limsup _{m \rightarrow \infty} C_{\rho_{\epsilon_{m}}} \leq \inf _{\xi \in \mathbb{R}^{n}} C(\xi),
\end{aligned}
$$

showing that $C\left(\xi^{*}\right)=\inf _{\xi \in \mathbb{R}^{n}} C(\xi)$.

Now we prove the convergence of $w_{\epsilon}$ as $\epsilon \rightarrow 0$.

Lemma 4.3. For every sequence $\left\{\epsilon_{m}\right\}$ there is a subsequence, we keep calling the same, so that $w_{\epsilon_{m}}=w_{m} \rightarrow w$ in $H^{\alpha}\left(\mathbb{R}^{n}\right)$, when $m \rightarrow \infty$, where $w$ is a solution of (4.7). 
Proof. Since $w$ is a solution of (4.7), from Lemma 3.3, we have

$$
\begin{aligned}
& \inf _{\xi \in \mathbb{R}^{n}} C(\xi)=C\left(\xi^{*}\right) \leq J_{\xi^{*}}(w)=J_{\xi^{*}}(w)-\frac{1}{2} J_{\xi^{*}}^{\prime}(w) w \\
& =\left(\frac{1}{2}-\frac{1}{p+1}\right) \int_{\mathbb{R}^{n}} K\left(\xi^{*}\right)|w|^{p+1} d x \\
& \leq\left(\frac{1}{2}-\frac{1}{p+1}\right) \liminf _{m \rightarrow \infty} \int_{\mathbb{R}^{n}} K\left(\epsilon_{m} x+\epsilon_{m} y_{\epsilon_{m}}\right)\left|w_{m}\right|^{p+1}(x) d x \\
& \leq\left(\frac{1}{2}-\frac{1}{p+1}\right) \limsup _{m \rightarrow \infty} \int_{\mathbb{R}^{n}} K\left(\epsilon_{m} x+\epsilon_{m} y_{\epsilon_{m}}\right)\left|w_{m}\right|^{p+1} d x \\
& =\left(\frac{1}{2}-\frac{1}{p+1}\right) \limsup _{m \rightarrow \infty} \int_{\mathbb{R}^{n}} K\left(\epsilon_{m} x\right)\left|v_{m}\right|^{p+1} d x \\
& \leq \limsup _{m \rightarrow \infty}\left(I_{\rho_{\epsilon_{m}}}\left(v_{m}\right)-\frac{1}{p+1} I_{\rho_{\epsilon_{m}}}^{\prime}\left(v_{m}\right) v_{m}\right) \\
& =\limsup _{m \rightarrow \infty} C_{\bar{\rho}_{\epsilon_{m}}} \leq \inf _{\xi \in \mathbb{R}^{n}} C(\xi) .
\end{aligned}
$$

The above estimates gives

$$
\lim _{m \rightarrow \infty} \int_{\mathbb{R}^{n}} K\left(\epsilon_{m} x+\epsilon_{m} y_{\epsilon_{m}}\right)\left|w_{m}\right|^{p+1} d x=\int_{\mathbb{R}^{n}} K\left(\xi^{*}\right)|w|^{p+1} d x .
$$

Consequently,

$$
\begin{aligned}
& \text { (a) } \lim _{m \rightarrow \infty} \int_{\mathbb{R}^{n}} \int_{\mathbb{R}^{n}} \frac{\left|w_{m}(x+z)-w_{m}(x)\right|^{2}}{|z|^{n+2 \alpha}} d z d x=\int_{\mathbb{R}^{n}} \int_{\mathbb{R}^{n}} \frac{|w(x+z)-w(x)|^{2}}{|z|^{n+2 \alpha}} d z d x \\
& \text { (b) } \lim _{m \rightarrow \infty} \int_{\mathbb{R}^{n}} Q\left(\epsilon_{m} x+\epsilon_{m} y_{\epsilon_{m}}\right)\left|w_{m}(x)\right|^{2} d x=\int_{\mathbb{R}^{n}} Q\left(\xi^{*}\right)|w(x)|^{2} d x .
\end{aligned}
$$

From (b), given $\delta>0$ there exists $R>0$ such that

$$
\int_{|x| \geq R} Q\left(\epsilon_{m} x+\epsilon_{m} y_{\epsilon_{m}}\right)\left|w_{m}(x)\right|^{2} d x \leq \delta .
$$

Furthermore, using $\left(H_{3}\right)$, we obtain

$$
\int_{|x| \geq R}\left|w_{m}(x)\right|^{2} d x \leq \frac{\delta}{a_{1}} .
$$

On the other hand

$$
\lim _{m \rightarrow \infty} \int_{|x| \leq R}\left|w_{m}(x)\right|^{2} d x=\int_{|x| \leq R}|w(x)|^{2} d x .
$$

From (4.8) and (4.9), $w_{m} \rightarrow w$ in $L^{2}\left(\mathbb{R}^{n}\right)$. From this, given $\delta>0$ there are $\epsilon_{0}, R>0$ such that

$\int_{B^{c}\left(x^{*}, \epsilon_{m} R\right)}\left|u_{\epsilon_{m}}\right|^{2} d x \leq \epsilon_{m}^{n} \delta \quad$ and $\quad \int_{B\left(x^{*}, \epsilon_{m} R\right)}\left|u_{\epsilon_{m}}\right|^{2} d x \geq \epsilon_{m}^{n} C, \quad \forall \epsilon_{m} \leq \epsilon_{0}$, where $C$ is a constant independent of $\delta$ and $m$, showing the concentration of solutions $\left\{u_{\epsilon_{n}}\right\}$. 


\section{REFERENCES}

[1] C.O. Alves and S.H.M. Soares, Existence and concentration os positive solutions for a class of gradient systems, Nonlinear Differential Equations Appl. 12, 437-457 (2005).

[2] C.O. Alves, S.H.M. Soares and J. Yang, On existence and concentration of solutions for a class of Hamiltonian Systems in $\mathbb{R}^{n}$, Advanced Non. Studies 3, 161-189 (2003).

[3] M. Cheng, Bound state for the fractional Schrödinger equation with undounded potential, J. Math. Phys. 53, 043507 (2012).

[4] G. Chen and Y. Zheng, Concentration phenomenon for fractional nonlinear Schrödinger equations, Comm. Pure Appl. Anal. 13(6), 2359 - 2376 (2014).

[5] J. Dávila, M. Del Pino and J. Wei, Concentrating standing waves for the fractional nonlinear Schrödinger equation, J. Differential Equations. 256, 858-892 (2014).

[6] S. Dipierro, G. Palatucci, E. Valdinoci, Existence and symmetry results for a Schrödinger type problem involving the fractional Laplacian, Matematiche 68, 201-216 (2013).

[7] E. DiNezza, G. Palatucci and E. Valdinoci, Hitchhiker's guide to the fractional Sobolev spaces. Bull. Sci. math. 136, 521-573 (2012).

[8] P. Felmer, A. Quaas and J. Tan, Positive solutions of nonlinear Schrödinger equation with the fractional laplacian, Proceedings of the Royal Society of Edinburgh: Section A Mathematics, 142, No 6, 1237-1262 (2012).

[9] P. Felmer and C. Torres, Non-linear Schrödinger equation with non-local regional diffusion. Calc. Var. Partial Diff. Equ. 54, 75-98 (2015).

[10] P. Felmer and C. Torres, Radial symmetry of ground states for a regional fractional nonlinear Schrödinger equation. Comm. Pure Appl. Anal. 13, 2395-2406 (2014).

[11] Q-Y. Guan, Integration by Parts Formula for Regional Fractional Laplacian. Commun. Math. Phys. 266, 289329 (2006).

[12] Q-Y. Guan, Z.M. Ma, The reflected $\alpha$-symmetric stable processes and regional fractional Laplacian. Probab. Theory Relat. Fields 134, 649694 (2006)

[13] H. Ishii and G. Nakamura, A class of integral equations and approximation of $p$ Laplace equations, Calc. Var. 37, 485-522 (2010).

[14] S. Secchi, Ground state solutions for nonlinear fractional Schrödinger equations in $\mathbb{R}^{n}$, J. Math. Phys. 54, 031501 (2013).

[15] X. Shang and J. Zhang, Concentrating solutions of nonlinear fractional Schrödinger equation with potentials, J. Differential Equations 258, 1106-1128 (2015).

[16] X. Shang and J. Zhang, Existence and multiplicity solutions of fractional Schrödinger equation with competing potential functions, Complex Variables and Elliptic Equations 61, 1435-1463 (2016).

[17] C. Torres, Symmetric ground state solution for a non-linear Schrödinger equation with non-local regional diffusion, Complex Variables and Elliptic Equations, http://dx.doi.org/10.1080/17476933.2016.1178730 (2016)

[18] C. Torres, Multiplicity and symmetry results for a nonlinear Schrödinger equation with non-local regional diffusion, Math. Meth. Appl. Sci. 39, 2808-2820 (2016).

[19] C. Torres, Nonlinear Dirichlet problem with non local regional diffusion, Fract. Cal. Appl. Anal. 19 2, 379-393 (2016).

[20] X. Wang and B. Zeng, On concentration of positive bound states of nonlinear Schrdinger equations with competing potential functions. SIAM J. Math. Anal. 28, 633-655 (1997). 